\title{
Non-capsulated and capsulated Haemophilus influenzae in children with acute otitis media in Venezuela: a prospective epidemiological study
}

\author{
Laura Naranjo ${ }^{1 *}$, Jose Antonio Suarez ${ }^{1}$, Rodrigo DeAntonio ${ }^{2}$, Francis Sanchez ${ }^{1}$, Alberto Calvo ${ }^{3}$, Enza Spadola ${ }^{4}$, \\ Nicolás Rodríguez ${ }^{3}$, Omaira Andrade ${ }^{3}$, Francisca Bertuglia ${ }^{3}$, Nelly Márquez ${ }^{3}$, Maria Mercedes Castrejon², \\ Eduardo Ortega-Barria ${ }^{5}$ and Romulo E Colindres ${ }^{6}$
}

\begin{abstract}
Background: Non-typeable Haemophilus influenzae (NTHi) and Streptococcus pneumoniae are major causes of bacterial acute otitis media (AOM). Data regarding AOM are limited in Latin America. This is the first active surveillance in a private setting in Venezuela to characterize the bacterial etiology of $\mathrm{AOM}$ in children $<5$ years of age.

Methods: Between December 2008 and December 2009, 91 AOM episodes (including sporadic, recurrent and treatment failures) were studied in 87 children enrolled into a medical center in Caracas, Venezuela. Middle ear fluid samples were collected either by tympanocentesis or spontaneous otorrhea swab sampling method. Standard laboratory and microbiological techniques were used to identify bacteria and test for antimicrobial resistance. The results were interpreted according to Clinical Laboratory Standards Institute (CLSI) 2009 for non-meningitis isolates. All statistical analyses were performed using SAS 9.1 and Microsoft Excel (for graphical purposes).

Results: Overall, bacteria were cultured from 69.2\% (63 of the 91 episodes); at least one pathogen (S. pneumoniae, H. influenzae, S. pyogenes or M. catarrhalis) was cultured from 65.9\% (60/91) of episodes. H. influenzae (55.5\%; 35/63 episodes) and S. pneumoniae (34.9\%; 22/63 episodes) were the most frequently reported bacteria. Among $\mathrm{H}$. influenzae isolates, 62.9\% (22/35 episodes) were non-capsulated (NTHi) and 31.4\% (11/35 episodes) were capsulated including types $\mathrm{d}, \mathrm{a}, \mathrm{c}$ and $\mathrm{f}$, across all age groups. Low antibiotic resistance for $\mathrm{H}$. influenzae was observed to amoxicillin/ampicillin (5.7\%; 2/35 samples). NTHi was isolated in four of the six $\mathrm{H}$. influenzae positive samples (66.7\%) from recurrent episodes.

Conclusions: We found H. influenzae and S. pneumoniae to be the main pathogens causing AOM in Venezuela. Pneumococcal conjugate vaccines with efficacy against these bacterial pathogens may have the potential to maximize protection against $\mathrm{AOM}$.
\end{abstract}

Keywords: Acute otitis media, Non-typeable Haemophilus influenzae (NTHi), Pneumococcal conjugate vaccine

\section{Background}

Acute otitis media (AOM) is one of the most frequently diagnosed bacterial infections in children following nasopharyngeal colonization [1]. It results in frequent pediatric visits both in the developed [2] and developing world [3]. Approximately, three in four children develop

\footnotetext{
* Correspondence: naranjo.laura1@gmail.com

'Policlínica Metropolitana, Piso 1, consultorio 1-13, Urbanización Caurimare, Calle A-1, Caracas, Venezuela

Full list of author information is available at the end of the article
}

at least one episode of AOM by 3 years of age, [4] with peak incidences observed among 6-18 month-old children [2]. A report on the epidemiology of AOM by Block and colleagues [5] showed that $64 \%$ of children had experienced their first episode by 6 months, and $86 \%$ by 1 year of age; $31 \%$ had $>3$ episodes in $<12$ months of age. AOM is, thus, one of the main causes of childhood morbidity in both developed and developing countries [6]. The estimated annual disease burden of AOM ranges between 8,200,000-12,900,000 cases among

\section{Biomed Central}


children $<5$ years of age in Latin America and the Caribbean [7]. In Venezuela, approximately 100,000-130,000 AOM cases per year (2004-2007) are reported in children $<5$ years of age (Ministerio del Poder Popular para la Salud. Gobierno Bolivariano de Venezuela, Personal communication, 2010).

AOM is a multi-pathogen disease [8]. Streptococcus pneumoniae (S. pneumoniae) and non-typeable Haemophilus influenzae (NTHi) have consistently been reported as the two major bacterial pathogens responsible for AOM, [9-11] identified in up to $80 \%$ of cases. Other regularly reported bacterial pathogens include Moraxella catarrhalis (M. catarrhalis) and Streptococcus pyogenes (S. pyogenes) [11]. S. pneumoniae has been shown to be responsible for $43.8 \%-61.4 \%$ of all bacterial AOM cases in Latin America [12-14]. H. influenzae has, however, emerged as the most frequently recovered bacterial pathogen in recent studies $[13,15]$. AOM caused by NTHi has been particularly associated with older age groups and recurrent disease [11]. Recurrent to chronic AOM may predispose to chronic suppurative otitis media (CSOM) in a third of cases [16]. Globally, 65-330 million people may suffer from CSOM [17].

Recurrent disease in children has important implications for AOM treatment. Although some cases resolve spontaneously, the majority require antibiotic treatment for early recovery. AOM constitutes the primary motive for the prescription of antibiotics in children [18]. The increasing use of these antibiotics may be acting as a selective pressure driving antibiotic resistance [19]. AOM is the primary cause of antibiotic consumption in Latin America and the Caribbean [20]. Antibiotic consumption in Latin America is highest in Argentina and Venezuela [21]. High prevalence of drug-resistant pathogens like penicillin-resistant $S$. pneumoniae and $\beta$-lactamase producing or other ampicillin-resistant $H$. influenzae have become major factors in complicating the management of AOM [22]. Efforts to prevent infectious diseases through vaccination may be able to reduce the demand for antibiotics in the community and the selective persistence of nasopharyngeal bacterial pathogens [23].

Pneumoccocal conjugate vaccines (PCVs) have been shown to be safe and effective in preventing invasive pneumococcal disease (IPD) in children [24,25]. The 7valent pneumococcal vaccine (PCV-7; Prevnar ${ }^{\mathrm{TM}} /$ Prevenar TM, Pfizer/Wyeth, US) was introduced into the US public immunization strategy in 2000 and has demonstrated noticeable efficacy against IPD [26,27]; 57\% (95\% CI: 44-67) efficacy against vaccine-type pneumococcal AOM [28] and overall 7\% (95\%CI: 4.1-9.7) efficacy against clinical AOM episodes [24]. Wide use of PCV-7 led to a relative shift in the proportion of pathogens causing persistent AOM was observed (decrease in $S$. pneumoniae and an increasing predominance of NTHi) [10]. Furthermore, with widespread vaccination, the predominant cause of remaining cases of pneumococcal disease became non-vaccine serotypes of $S$. pneumoniae $[29,30]$, due to changing antimicrobial resistance patterns and secular trends. There are two new PCVs licensed recently in Venezuela. The 10-valent pneumococcal $/ H$. influenzae protein D conjugated vaccine (PHiD-CV, Synflorix ${ }^{\mathrm{TM}}$, GlaxoSmithKline [GSK] Biologicals, Rixensart, Belgium) has 8 of the 10 serotypes conjugated to a recombinant form of protein $\mathrm{D}$ of $H$. influenzae. A randomized, double-blind study with an 11 -valent prototype vaccine relying on the protein $\mathrm{D}$ carrier provides evidence that proteinD-containing vaccines may offer additional protection (35\%) against AOM episodes caused by both pneumococcus and $H$. influenzae [31]. A 13-valent pneumococcal vaccine formulation (PCV-13) is also available.

In Venezuela, Haemophilus influenzae type $B$ was introduced into the expanded program of immunization in 2000 [32]. Pneumoccocal conjugate vaccines are not indicated for universal vaccination, however since 2004, PCV-7 has been gradually introduced into private practice. Data on AOM in Latin America are limited and no there are no data on AOM etiology in Venezuela. This study thus provides novel data on the bacterial etiology of AOM and the associated serotypes in Venezuelan children. This study assessed the microbiology of AOM cases, antibiotic susceptibility pattern of the middle ear pathogens obtained from these cases, and the proportion of AOM episodes that may be vaccine-preventable with new pneumococcal vaccines recently licensed.

\section{Methods}

\section{Study design and subjects}

We conducted a prospective epidemiological study within a routine, private clinical setting in a medical center in Caracas, Venezuela from December 2008 to December 2009. Children $\geq 3$ months and $<5$ years of age, visiting physician/pediatrician clinics for AOM and for whom a MEF sample was available, were enrolled in the study. We included children presenting with one of the general signs of otalgia/irritability, conjunctivitis, fever and either Paradise's criteria (bulging, diffused or localized inflamed tympanic membranes) or spontaneous otorrhea $(<24 \mathrm{~h})$. Exclusion criteria included: children hospitalized at the time of the diagnosis or treatment of AOM, presenting with a new episode of AOM but having received antibiotic treatment in the previous $72 \mathrm{~h}$ for a different illness, those who had received antibiotic by the physician/ear, nose and throat (ENT) specialist prior to tympanocentesis and those with otorrhea for $>24$ h or with otitis media with effusion (not AOM). 
Two specific groups of children were eligible for study recruitment: - Children with a new episode of AOM (< $72 \mathrm{~h}$ of onset) who were yet to receive antibiotic therapy for the episode, and - Children with a diagnosis of AOM within 48-72 h before study enrolment, who had received antibiotic therapy, but remained symptomatic at the time of study entry (treatment failures).

A child could be enrolled multiple times in the study whenever he/she reported a new episode. A case was considered as a new episode if there was a 30-day symptom-free interval since the resolution of the first episode. A recurrent AOM episode was the third or greater new episode within the last 6 months or the fourth or greater new episode within the last 12 months.

The primary endpoints were occurrence of $H$. influenzae, S. pneumoniae, M. catarrhalis and S. pyogenes isolated from MEF samples. Secondary endpoints were occurrence of $H$. influenzae and S. pneumoniae specific serotypes from MEF samples and antimicrobial susceptibility of all bacterial pathogens isolated from MEF samples.

Written informed consent was obtained from the parents/guardians of participating children before conducting any study-related procedures. We collected demographic information including age and sex assuring confidentiality for subjects enrolled.

\section{Bacterial identification and characterization}

Samples of MEF from all children were collected by an ENT specialist by performing tympanocentesis or by careful sampling of spontaneous otorrhoea (removal and cleaning of the ear canal material and deep aspiration of the MEF material via needle insertion).

\section{Microbiology}

MEF samples were inoculated into Amies transport medium with charcoal [33], sent to the study laboratory (Laboratorio Metropolitano) within 16-48 h, inoculated onto chocolate and blood agar and incubated at $37^{\circ} \mathrm{C}$ in a $5 \% \mathrm{CO}_{2}$ atmosphere. For identification, standard guidelines described by the American Society of Microbiology were used and confirmed by using $\mathrm{NH}$ and GP cards VITEK-2 compact system (bioMérieux ${ }^{\mathrm{TM}}$ ).

\section{Clinical feature}

A detailed medical history including symptoms, seasonality, vaccination status for PCV and $\mathrm{HiB}, \mathrm{AOM}$ episodes characteristics including recurrency, A clinical examination was performed recording all data in the study database.

\section{Antibacterial susceptibility}

Antimicrobial susceptibility was done using AST-P68 (bioMérieux $^{\mathrm{TM}}$ ) for S. pneumoniae, standard methods for $H$. influenzae and $M$. catarrhalis were according to Clinical Laboratory Standards Institute (CLSI-M100S19) 2009 and for quality control, S. pneumoniae ATCC 49619 and $H$. influenzae ATCC 49247 y 49766 [34] strains were used. Serotyping for S. pneumoniae was done at the national reference laboratory, Laboratorio de Microbiologia del Instituto Nacional de Higiene "Rafael Rangel" using Quellung reaction following SIREVA II guidelines [35]. $H$. influenzae isolates were confirmed by polymerase chain reaction (PCR) procedures and $\beta$-lactamase testing (nitrocefin disk) was performed.

\section{Statistical analyses}

We performed the analyses only on the ATP cohort (subjects meeting all eligibility criteria, complying with the protocol-defined procedures, with no elimination criteria during the study and for whom laboratory results of the MEF samples was available). The study results were presented as episodes of AOM.

We calculated the proportions of AOM caused by $S$. pneumoniae, $H$. influenzae and other bacterial pathogens. Distribution analyses of $S$. pneumoniae and $H$. influenzae serotypes by age, procedure and pneumococcal vaccination status according to age (fully vaccinated, partially vaccinated and unvaccinated) for AOM cases were performed. We also determined seasonality of AOM cases and other isolates. Qualitative variables, including symptoms and vaccination, were compared to explore differences among capsulated and non capsulated $H$. influenzae status with the Fisher exact test. All tests were 2 sided, and the level of significance was set at $\mathrm{p}<0.05$.

All statistical analyses were performed using Statistical Analysis System (SAS) version 9.1 (or higher) and Microsoft Excel (for graphical purposes).

\section{Ethics}

The study was conducted according to Good Clinical Practice guidelines, the Declaration of Helsinki and the local rules and regulations of the country. All the studyrelated documents were reviewed and approved by the local Independent Ethics Committee/Institutional Review Board and the study adhered to applicable local guidelines.

\section{Results}

\section{Demographic characteristics and sample description}

We collected data on 92 episodes of AOM reported from 87 enrolled children. Ninety-one episodes were retained in the ATP (according-to-protocol) cohort due to one child being enrolled more than once for the same AOM episode. Only one of these episodes was classified as a treatment failure. Of the 87 children included in the ATP cohort, 84 reported a single 
episode, two reported two episodes and one child reported three episodes. Any consecutive episode occurred with an interval of $>30$ days after the previous episode.

The mean age of the children for all episodes was 28.3 months; the proportion of females was 51.6\% (47/91 episodes) (Additional file 1: Table S1).

The majority of the episodes $(57.2 \% ; 52 / 91)$ were reported in the 1-3 years age group; $30.8 \%$ (28/91 episodes) and $26.4 \%$ (24/91 episodes) occurred in the $12-23$ months and 24-35 months age groups, respectively.

We collected one MEF (middle ear fluid) sample from each of the 91 episodes. Most samples (90.1\%; 82/91 episodes) were collected by tympanocentesis, while nine samples $(9.9 \%)$ were collected from spontaneous otorrhea episodes. Of the nine episodes with spontaneous otorrhea, $18.2 \%(2 / 11), 17.9 \%(5 / 28)$ and $8.3 \%(2 / 24)$ were distributed in the 3-11 months, 12-23 months and 24-35 months age groups, respectively.

\section{Bacterial etiology of episodes}

We observed pathogenic bacterial growth in 67.1\% (55/ 82 episodes) and $88.9 \%$ (8/9 episodes) of samples collected by tympanocentesis and spontaneous otorrhoea, respectively. Overall, bacteria were cultured from $69.2 \%$ (63) of the 91 episodes; one treatment failure episode of AOM did not show any bacterial growth. Overall, 65.9\% (60) of the episodes yielded positive cultures for at least one of the four pathogenic bacteria. The most commonly isolated bacteria among MEF samples with bacterial growth were $H$. influenzae and S. pneumoniae, detected in 55.5\% (35/63) and 34.9\% (22/63) of episodes, respectively. Only $3.2 \%(2 / 63)$ episodes were positive for $S$. pyogenes, and $1.6 \%$ (1/63 episodes) for $M$. catarrhalis. No single episode was positive for both $S$. pneumoniae and $H$. influenzae.

The highest number of positive episodes for at least one of the four pathogenic bacteria under study was in the $3-11$ months age group $(72.7 \% ; 8 / 11$ episodes); the lowest number of positive episodes was found in the 2435 months age group (58.3\%; 14/24 episodes). The most frequently isolated bacteria across all age groups were H. influenzae and S. pneumoniae (Figure 1).

Among episodes with spontaneous otorrhea, S. pneumoniae was isolated in three episodes, S. pyogenes in two, $H$. influenzae in one episode; the remaining two episodes corresponded to other agents.

Recurrent AOM episodes were reported for 5.4\% (14/ 91 episodes) during the study. We obtained bacterial cultures from samples from $71.4 \%(10 / 14)$ of these recurrent episodes. Of these, 6/10 were positive for $H$. influenzae; 3/10 for S. pneumoniae and 1/10 was positive for $M$. catarrhalis.

\section{Bacterial etiology by serotype}

Most $H$. influenzae strains were non-capsulated (NTHi: $62.9 \% ; 22 / 35$ episodes). This was followed by serotype $d$ (17.1\%; 6/35 episodes), $a$ and $c$ (each accounting for $5.7 \% ; 2 / 35$ episodes), and $f(2.9 \% ; 1 / 35$ episodes $)$. Two samples $(5.7 \% ; 2 / 35$ episodes) were reported as unknown serotype.

Serotype 19A was the most frequently isolated $S$. pneumoniae serotype (40.9\%; 9/22 episodes) followed by serotypes 6A, 11 and 15 (each accounting for 9.1\%; 2/22 episodes), and $23 \mathrm{~F}$ and $7 \mathrm{C}$ (each accounting for $4.5 \%$; $1 / 22$ episodes). Five samples (22.7\%; 5/22 episodes) were reported as unknown (Figure 2).

A similar trend of serotype distribution was observed across the age groups for both $H$. influenzae and $S$. pneumoniae.

Of the six $H$. influenzae positive samples from recurrent episodes, NTHi was isolated in four samples (66.7\%; 4/6 samples). The remaining two samples corresponded to types c and a. The three $S$. pneumoniae positive samples from recurrent episodes corresponded to serotypes $7 \mathrm{C}, 23 \mathrm{~F}$ and one was reported as unknown. Of the 14 recurrent AOM episodes, the highest numbers of episodes were reported in the 24-35 months and 36-47 months age groups (5 episodes each); three episodes were reported in the 12-23 months and one episode in the 3-11 months age groups.

\section{Common symptoms reported}

Fever (65.9\%; 60/91 episodes) and ear pain (57.1\%; 52/91 episodes) were the most common symptoms reported before the study visit and also during the study visit (49.5\%; 45/91 episodes and 56\%; 51/91 episodes, for fever and ear pain respectively). We did not observe significant differences between capsulated and non-capsulated $H$. influenzae for fever $(68.2 \%$ vs. $72.7 \% ; p=0.78)$ and pain ( $45.5 \%$ vs. $54.5 \%$; $p=0.62)$.

\section{Seasonal distribution of bacterial etiology of episodes}

AOM episodes occurred throughout the year; the highest numbers of episodes were recorded for children enrolled in 2009 (July: 14 episodes; March: 10 episodes). The highest number of $H$. influenzae positive episodes was in children enrolled in April and July (14.3\% each; $5 / 35$ episodes). At least one $S$. pneumoniae positive episode was reported for most months of the year between January and April, and July and December, inclusive, the highest number of episodes being enrolled in July (22.7\%; 5/22 episodes) (Figure 3).

\section{Vaccination status}

About $80.2 \%$ (73/91 episodes) of episodes occurred in children who received at least one dose of the PCV-7. 


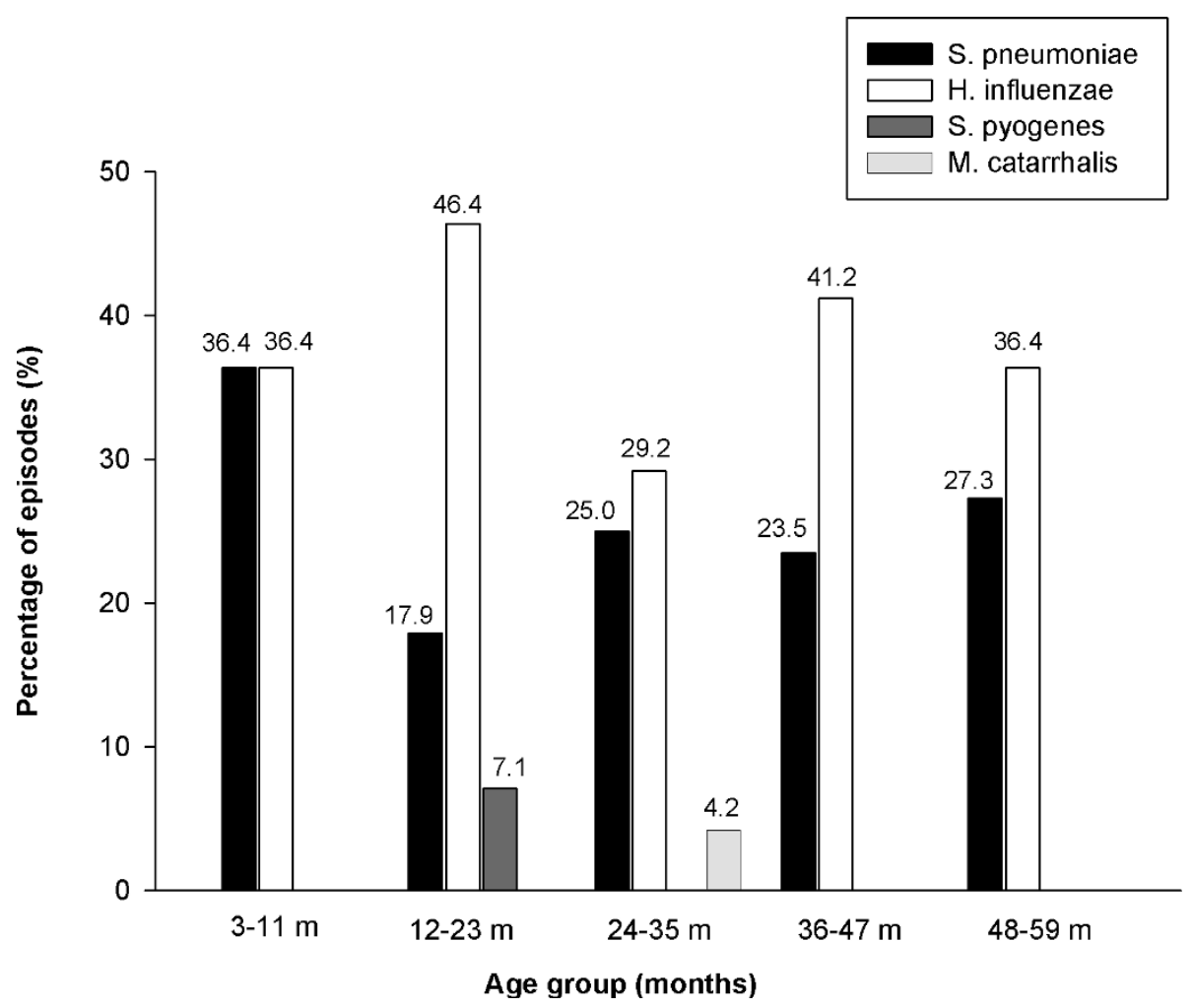

Figure 1 Bacterial etiology of episodes by age (ATP Cohort). The simple bars represent the percentage of bacterial episodes in each age group.

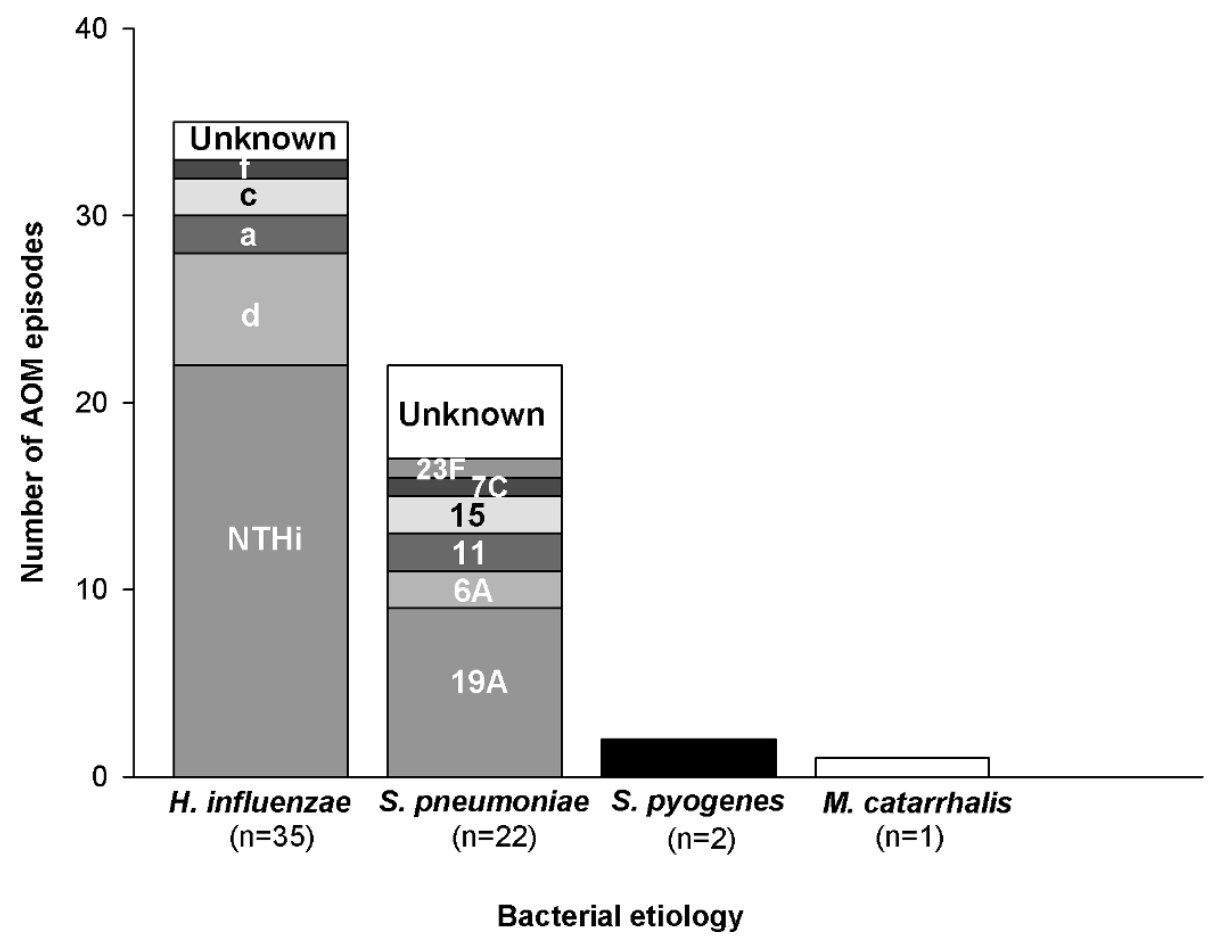

Figure 2 Bacterial etiology by serotypes (ATP Cohort). The stacked column bars represent the number of serotypes for each bacterial positive episode. 


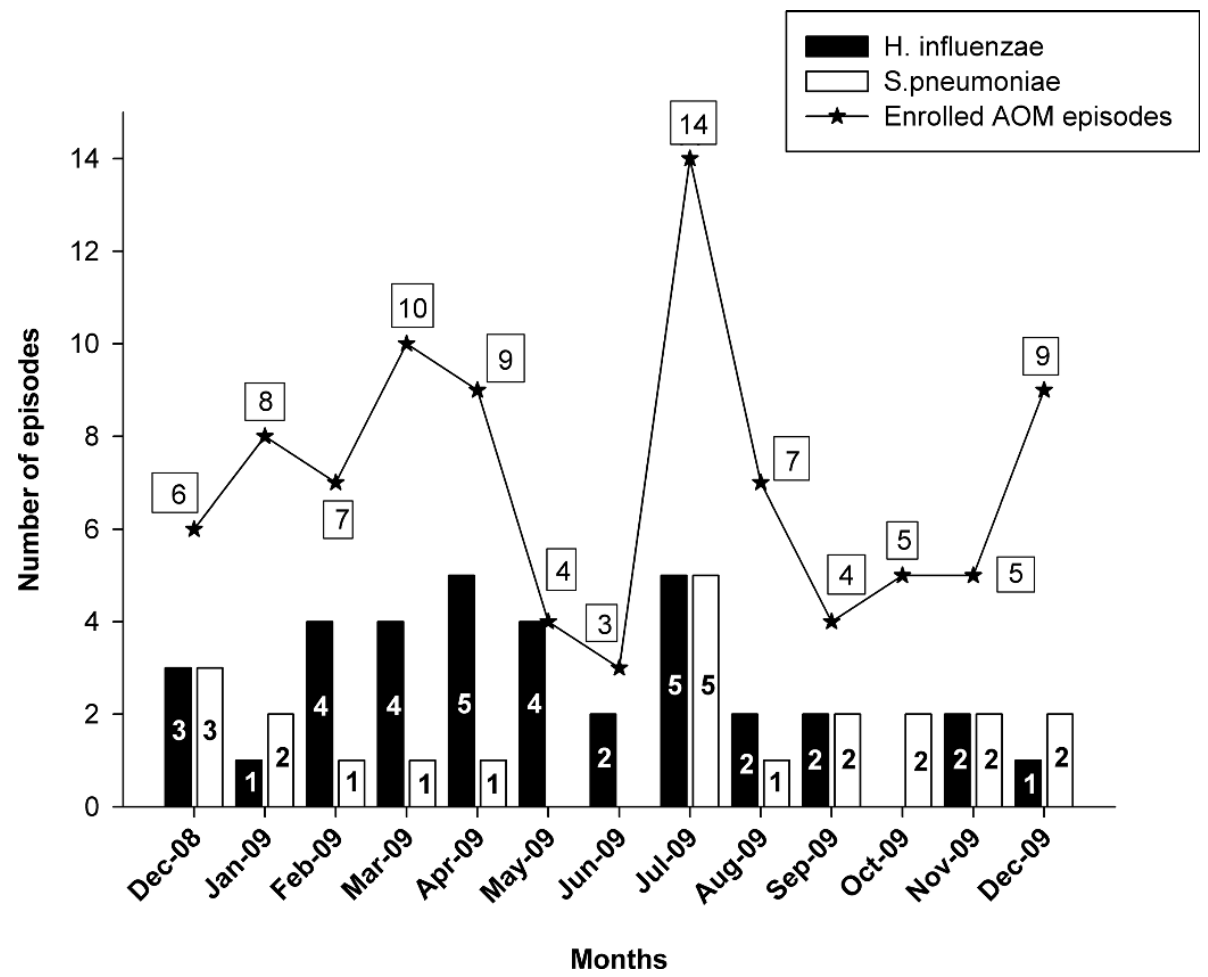

Figure 3 Seasonal distribution of AOM episodes (ATP Cohort). The blue stacked line graph shows the number of AOM episodes enrolled in each month and the simple bar graph shows the number of $\mathrm{H}$. influenzae and $\mathrm{S}$. pneumoniae episodes isolated each month.

About 97.1\% (34/35) subjects testing positive for $H$. influenzae received at least one $H$. influenzae type $b$ vaccine dose.

The percentage of episodes with positive cultures for at least one of the four study bacteriawas $69.6 \%(32 / 46$ episodes) in fully vaccinated children, $63 \%$ (17/27 episodes) in partially vaccinated and $57.1 \%$ (8/14 episodes) in unvaccinated children.

More specifically for S. pneumoniae, the percentage of culture positive episodes was $30.3 \%$ (10/33 episodes), $35.3 \%$ (6/17 episodes) and $40.0 \%$ (4/10 episodes) in the fully vaccinated, partially vaccinated and unvaccinated children, respectively. The percentage of $H$. influenzae culture positive episodes were $63.6 \%$ (21/33 episodes) in the fully vaccinated children, $52.9 \%$ (9/17 episodes) in partially vaccinated and $40.0 \%$ (4/10 episodes) in unvaccinated children, respectively. We did not find significant differences between capsulated and non-capsulated $H$. influenzae or between fully vaccinated and partially/ unvaccinated groups $(p=0.38)$.

\section{Antibacterial susceptibility}

Among the $35 \mathrm{H}$. influenzae positive samples, 94.3\% (33/35) were sensitive to amoxicillin/ampicillin, 5.7\% (2/

$35)$ were resistant to amoxicillin/ampicillin and 2.9\% (1/

35 ) showed intermediate resistance to cefuroxime. $25.7 \%$
(9/35) samples showed resistance to trimethoprim/sulfamethoxazole. The two isolates resistant to amoxicillin/ ampicillin and susceptible to amoxicillin/clavulanate were tested for $\beta$-lactamase activity with positive results $(\mathrm{n}=2)$.

Among the 22 S. pneumoniae positive samples, $77.3 \%$ $(17 / 22)$ were sensitive, $18.2 \%(4 / 22)$ and $4.5 \%(1 / 22)$ of samples were intermediate and resistant to penicillin, respectively (Figure 4). All S. pneumoniae isolates tested except three were sensitive to amoxicillin and ampicillin $(86.4 \% ; 19 / 22)$. Erythromycin resistance was reported in $33.3 \%(7 / 22)$ samples; $35 \%(7 / 22)$ of samples were resistant to tetracycline and $45.5 \%(10 / 22)$ to trimethoprim/ sulfamethoxazole.

Of the nine samples positive for S. pneumoniae serotype $19 \mathrm{~A}, 77.8 \%$ (7/9) were sensitive to penicillin and intermediate resistance was observed for $22.2 \%(2 / 9)$ samples. No resistance to penicillin was noted for this serotype. No antibiotic resistance was observed to cefotaxime, chloramphenicol or levofloxacin.

\section{Discussion}

This is the first study to investigate the bacterial etiology of AOM in a private setting in Venezuela. Our findings are novel in that we identified non-capsulated and capsulated $H$. influenzae as the main pathogens causing 


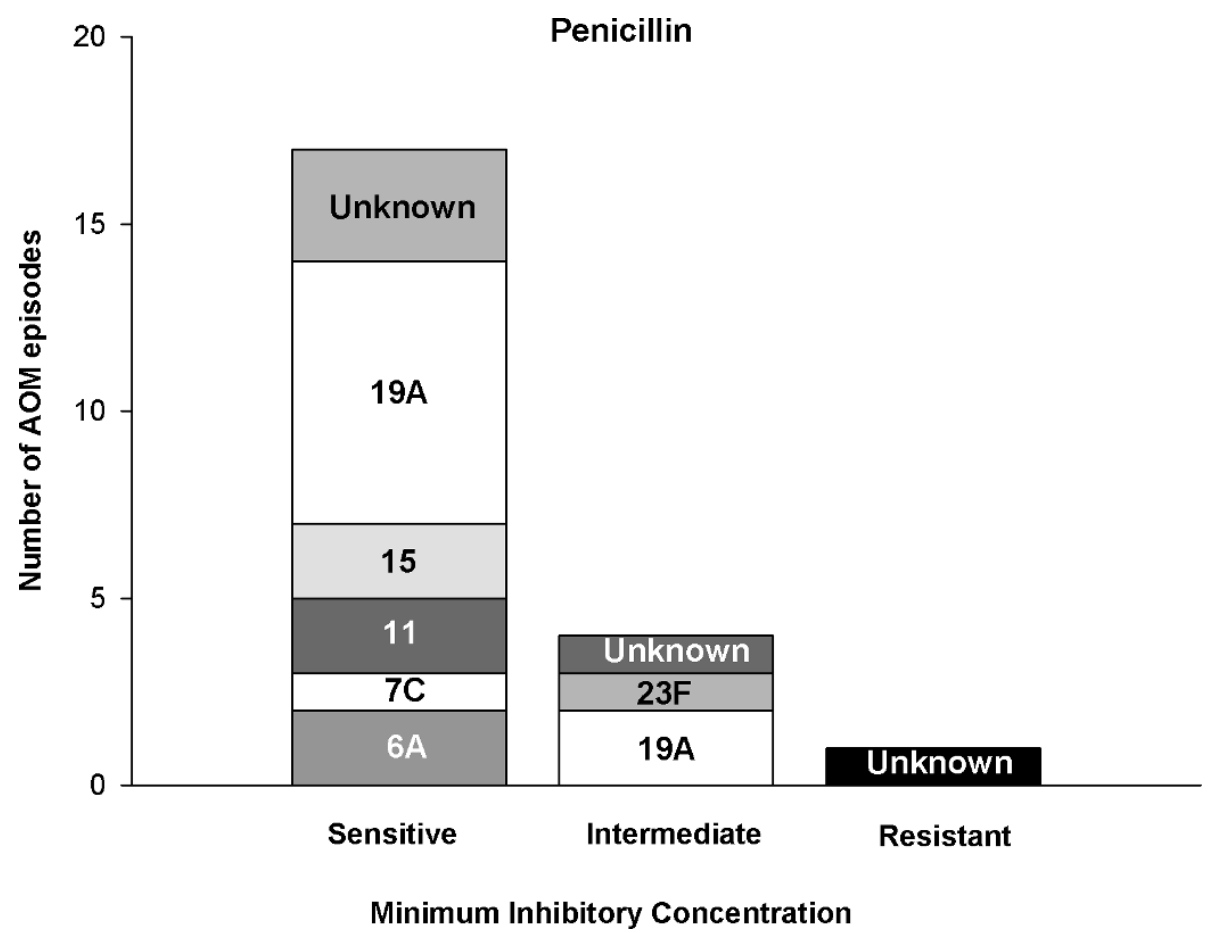

Figure 4 Penicillin resistance for S. pneumoniae isolates (ATP Cohort). Susceptibility of each S. pneumoniae serotype to penicillin for categories viz; sensitive, intermediate and resistant.

AOM in Venezuela. Among the episodes reported, $H$. influenzae (55.5\%) and S. pneumoniae (34.9\%) were the most commonly isolated bacteria. This is in-line with other studies who have reported these bacteria to be frequently isolated in other Latin American countries including Argentina [36], Chile [13], Costa Rica [12,37], Mexico [38] and Colombia [39]. Following the introduction of PCV-7 and the inclusion of the vaccine into the universal immunization program in the United States, a significant decrease in the proportion of S. pneumoniae and an increase in $H$. influenzae proportion among MEF isolates have been reported (2003-2006) [40]. A similar pattern was observed in the present study and has been observed in previous studies $[10,41]$ following introduction of the PCV. An increase in the occurrence of nonPCV-7 S. pneumoniae serotypes has also been described previously [42].

In the current analysis, we show that $H$. influenzae isolated in Venezuelan children was most commonly NTHi (62.9\%). NTHi has previously been reported to be associated with a history of recurrent AOM episodes, treatment failure and AOM within two weeks of completing a course of any antibiotic [43]. The high prevalence of NTHi in this private setting could be explained by the fact that vaccine coverage for PCV was considerably high (63.6\%). In this previous study, the authors showed that NTHi was not only the most prevalent cause of recurrent episodes of AOM but also of new cases and was detected across all age groups [44]. This finding differs from earlier reports of NTHi in children 2 years or older [11]. In our present study, most of the episodes we reported were in children younger than 24 months; NTHi was the most prevalent type among all age groups, including 3-11 month olds, who showed an equal distribution with $S$. pneumoniae.

We also report the isolation of capsulated $H$. influen$z a e$ including types $d, c, a$ and $f$, which has not been reported previously. These were not only isolated in all age groups, but also reported in recurrent episodes. In a recent systematic review for Latin America, capsulated types were not confirmed in MEF samples from AOM etiology studies except for $b$ type. This was also consistent with the results from other studies in Europe, such as France, where $H$. influenzae isolates were identified as NTHi. This could be explained by the fact that nasopharyngeal colonization of encapsulated $H$ influenzae is uncommon, occurring only in $2-5 \%$ of children $[45,46]$

We found serotype 19A to be the most commonly isolated S. pneumoniae serotype (40.9\%) from children who were fully vaccinated with the $P C V$, suggesting that PCV-7 vaccination does not offer a high level of crossprotection against 19A. Recently, in the US and other countries the non-vaccine serotype 19A has been associated with an increase in the proportions of IPD and 
AOM $[10,26,28,41,47]$. Other less commonly found bacterial pathogens in this present study were $S$. pyogenes (2.2\%) and M. catarrhalis (1.1\%), similar to other studies $[36,48]$.

We observed a seasonal pattern of AOM in the present study that was similar to that observed in a recent study in Colombia [39]. We found that AOM episodes were reported throughout the year, with the highest number of episodes reported in July, corresponding to the rainy season in Venezuela.

An increased use of antibiotics has been observed in Latin American countries, predominantly in Venezuela [21]. Antibiotic resistance to S. pneumoniae and $H$. influenzae has become a serious public health issue, more so in Latin America due to increased S. pneumoniae resistance to penicillin and other antibiotics [7]. Seven of nine S. pneumoniae 19A isolates were sensitive to penicillin in our study, highlighting the low prevalence of penicillin-resistant serotype 19A. In the $H$. influenzae isolates reported, $25.7 \%$ were resistant to trimethoprim-sulfamethoxazole and $5.7 \%$ to amoxicillin/ ampicillin. We did not observe antibiotic resistance to amoxicillin/clavulanate, cefotaxime, chloramphenicol and tetracycline. A limited occurrence of amoxicillin resistance has been previously detected in another multinational study [49]. Hence, amoxicillin and amoxicillin/clavulanate continue to be considered as appropriate treatment options for AOM in children [50]. Reducing antibiotic use by maximizing vaccination uptake may be a useful strategy to eradicate antibiotic-resistant serotypes.

PCVs could be an important tool in reducing the AOM burden. Besides offering protection against the vaccine serotypes, previous studies suggest that PCV7CRM provides cross-protection against the non-vaccine serotype $6 \mathrm{~A}$ for IPD and AOM $[28,51]$ and that PCVs with different conjugation methods or carrier proteins may provide modest (though non-significant) protection against 19A disease [52]. Antibiotic resistance and selection pressures are also likely to have an impact on the efficacy of PCVs. Vaccines that may protect against non-capsulated and capsulated $H$. influenzae serotypes may also play an important role to reduce the number of AOM episodes. Efficacies of $35.6 \%$ and $35.3 \%$ have previously been shown [31] against all $H$. influenzae and NTHi otitis media episodes with a prototype pneumococcal vaccine conjugated to the $H$ influenzae protein $\mathrm{D}$. This protein is present in capsulated and non-capsulated isolates and hence may potentially protect against AOM episodes caused by these agents. The proportions of pathogens causing $\mathrm{AOM}$ and potentially targeted by each vaccine formulation would be $55 \%$ for PHiD-CV and $19 \%$ for PCV-13. However, it is important to note that no efficacy or effectiveness data exist for either formulation. Additionally, previous studies with the precursor vaccines (PCV-7; 11-valent pneumococcal protein $\mathrm{D}$ conjugate $\mathrm{PHiD}-\mathrm{CV}$ predecessor vaccine) have demonstrated efficacy of $<100 \%$ against the targeted pathogens $[28,31]$. The precise magnitude of protection is therefore unknown.

This study is limited by a small sample size which affects the precision of the estimates. In addition, the enrolled children were recruited from a private center and thus may not truly represent the Venezuelan population. This is particularly important given the potential differences in exposure and risk factors for otitis media across the country.

\section{Conclusions}

In summary, this was the first active surveillance for $\mathrm{AOM}$ etiology in Venezuela in a private setting in a highly PCV vaccinated population. NTHi was the most prevalent etiological agent, suggesting that protection against NTHi is crucial for AOM prevention. Reduced antibiotic use and vaccine with efficacy against $H$. influenzae (beyond type b) and S. pneumoniae could be effective strategies for preventing AOM disease among children in Venezuela.

\section{Additional material}

Additional file 1: Table S1. Demographic characteristics with bacterial etiology of episodes by age and gender (ATP Cohort).

\section{Abbreviations}

AOM: acute otitis media; ATP: according-to-protocol; Cl: confidence interval; CLSI: Clinical and Laboratory Standards Institute; CSOM: chronic suppurative otitis media; ENT: Ear: nose: throat; GSK: GlaxoSmithKline; $H$. influenzae: Haemophilus influenzae; IPD: invasive pneumococcal disease; M. catarrhalis: Moraxella catarrhalis; MEF: middle ear fluid; NTHi: Non-typeable Haemophilus influenzae; PCR: polymerase chain reaction; PCV: pneumococcal conjugate vaccine; PHiD-CV: pneumococcal non-typeable Haemophilus influenzae protein-D conjugate vaccine; S. aureus: Staphylococcus aureus; S. pneumoniae: Streptococcus pneumoniae; S. pyogenes: Streptococcus pyogenes; SAS: Statistical Analysis System

\section{Acknowledgements}

Financial support was provided by GlaxoSmithKline, Inc. GSK (Latin America). The authors would like to thank the infants and their families for participating in this study. They also wish to thank Dr. Elizabeth Paradzik and Yorane González for providing their expertise as ENT specialists, Dr. Zaira Ron for study coordination, to all pediatricians from Policlínica Metropolitana for referring subjects for this study, and the GSK CARICAM team (Marianela Rojas, Helena Guerra and Diana Campo) for contributing and supporting the co-ordination and monitoring activities for this study. The authors would also like to acknowledge Mohammed Najeeb Ashraf for providing technical assistance in the preparation of this manuscript, Dr. Camilo Moreno and Jessica Mattos for management and coordination of this publication and Gunasekaran Ramakrishnan for helping with the statistical analysis (all employed by GSK Biologicals).

Synflorix is a trademark of the GlaxoSmithKline group of companies; Prevnar/ Prevenar is a trademark of Pfizer/Wyeth, USA. 


\section{Author details}

'Policlínica Metropolitana, Piso 1, consultorio 1-13, Urbanización Caurimare, Calle A-1, Caracas, Venezuela. ${ }^{2}$ GlaxoSmithKline Biologicals, Clayton, Ciudad del Saber Edificio 230, Panama City, Panama. ${ }^{3}$ Laboratorio Metropolitano, Sotano 2, Urbanización Caurimare, Calle A-1, Caracas, Venezuela. ${ }^{4}$ Instituto Nacional de Higiene, Ciudad Universitaria Los Chaguaranos, Caracas, Venezuela. ${ }^{5} \mathrm{GlaxoSmithKline} \mathrm{Biologicals,} \mathrm{Estrada} \mathrm{dos} \mathrm{Bandeirantes,} 8.464$ Jacarepaguá 22783-110 Rio de Janeiro, Brazil. ${ }^{6}$ GlaxoSmithKline Biologicals, Avenue Fleming 20, B-1300 Wavre, Belgium.

\section{Authors' contributions}

LN and JAS were involved in all processes related to enrolling patients, contributed to study design, interpretation of data, review and comments on all drafts of this paper and gave final approval to submit for publication. FS was the ENT who performed all tympanoscentesis, interpretation of data, review and comments on all drafts of this paper and gave final approval to submit for publication. AC, ES, NR, OA, FB and NM, worked actively in isolation and serotyping all samples, interpretation of data, review and comments on all drafts of this paper and gave final approval to submit for publication. RDA, EOB, REC and MMC contributed to study design, interpretation of data, review and comments on all drafts of this paper and gave final approval to submit for publication.

\section{Competing interests}

$L N$ and JAS received honoraria/paid expert testimony/travel grants from the commercial entity that sponsored the study.

MMC, RDA, EO-B and REC are employees of GlaxoSmithKline Biologicals. $E O B$ and REC have stock ownership; MMC, and RDA have stock options. $F S, A C, E S, N R, O A, F B$ and NM: No conflict.

Received: 28 September 2011 Accepted: 15 February 2012 Published: 15 February 2012

\section{References}

1. Centers for Disease Control and Prevention (CDC): Pneumococcal diseases in children-Q\&A.[http://www.cdc.gov/].

2. Klein JO: Otitis media. Clin Infect Dis 1994, 19:823-832

3. Berman S: Otitis media in developing countries. Pediatrics 1995, 96:126-131.

4. Cripps AW, Otczyk DC, Kyd JM: Bacterial otitis media: a vaccine preventable disease? Vaccine 2005, 23:2304-2310.

5. Block SL, Harrison CJ, Hedrick J, Tyler R, Smith A, Hedrick R: Restricted use of antibiotic prophylaxis for recurrent acute otitis media in the era of penicillin non-susceptible Streptococcus pneumoni. Int I Pediatr Otorhinolaryngol 2001, 61:47-60.

6. Brobby GW: The discharging ear in the tropics: a guide to diagnosis and management in a district hospital. Trop Doct 1992, 22:10-13.

7. Valenzuela MT, de Quadros C: Antibiotic resistance in Latin America: A cause of alarm. Vaccine 2009, 27(Suppl 3):25-28.

8. Segal N, Leibovitz E, Dagan R, Leiberman A: Acute otitis media-diagnosis and treatment in the era of antibiotic resistant organisms: updated clinical practice guidelines. Int J Pediatr Otorhinolaryngol 2005, 69:1311-1319.

9. Arguedas A, Sher L, Lopez E, Sáez-Llorens X, Hamed K, Skuba K, Pierce PF: Open label, multicenter study of gatifloxacin treatment of recurrent otitis media and acute otitis media treatment failure. Pediatr Infect Dis $J$ 2003, 22:949-956.

10. Casey JR, Pichichero ME: Changes in frequency and pathogens causing acute otitis media in 1995-2003. Pediatr Infect Dis J 2004, 23:824-828,

11. Leibovitz E, Jacobs MR, Dagan R: Haemophilus influenza: a significant pathogen in acute otitis media. Pediatr Infect Dis J 2004, 23:1142-1152.

12. Arguedas A, Dagan R, Soley C, Loaiza C, Knudsen K, Porat N, Perez A, Brilla E, Herrera ML: Microbiology of otitis media in Costa Rican children, 1999 through 2001. Pediatr Infect Dis J 2003, 22:1063-1068

13. Rosenblut A, Santolaya ME, González P, Corbalán V, Avendanõ LF, Martínez MA, Hormazabal JC: Bacterial and viral etiology of acute otitis media in Chilean children. Pediatr Infect Dis J 2001, 20:501-507.

14. Rosenblut A, Santolaya ME, Gonzalez P, Borel C, Cofre J: Penicillin resistance is not extrapolable to amoxicillin resistance in Streptococcus pneumonia isolated from middle ear fluid in children with acute otitis media. Ann Otol Rhinol Laryngol 2006, 115:186-190.
15. Pichichero ME: Evolving shifts in otitis media pathogens: relevance to a managed care organization. Am J Manag Care 2005, 11 (Suppl 6):192-201.

16. Homoe P, Christensen RB, Bretlau P: Acute otitis media and age at onset among children in Greenland. Acta Otolaryngol 1999, 119:65-71.

17. World Health Organization: Chronic suppurative otitis media: burden of illness and management options 2004.[http://www.who.int/pbd/deafness/ activities/hearing_care/otitis_media.pdf].

18. Al P, Rovers MM, Schilder AG, Verheij TJ, Hak E: Trends in doctor consultations, antibiotic prescriptions, and specialist referrals for otitis media in children: 1995-2003. Pediatrics 2006, 117:1879-1886.

19. Goossens H, Ferech M, Vander Stichele R, Elseviers M, ESAC Project Group: Outpatient antibiotic use in Europe and association with resistance: a cross-national database study. Lancet 2005, 365:548-549.

20. Constenla D, Gomez E, de la Hoz FP, O' Loughlin R, Sinha A, Valencia JE, Valenzuela MT: The burden of pneumococcal disease and costeffectiveness of a pneumococcal vaccine in Latin America and the Caribbean.[http://www.sabin.org/news-resources/publication/burdenpneumococcal-disease-and-cost-effectiveness-pneumococcal-vaccine-].

21. Wirtz VJ, Dreser A, Gonzales R: Trends in antibiotic utilization in eight Latin American countries, 1997-2007. Rev Panam Salud Publica 2010, 27:219-225.

22. Yamanka N, Hotomi M, Billal DS: Clinical bacteriology and immunology in acute otitis media in children. $J$ Infect Chemother 2008, 14:180-187.

23. Whitney CG, Klugman KP: Vaccines as tools against resistance: the example of pneumococcal conjugate vaccine. Semin Pediatr Infect Dis 2004, 15:86-93

24. Black S, Shinefield H, Fireman B, Lewis E, Ray P, Hansen JR, Elvin L, Ensor KM, Hackell J, Siber G, Malinoski F, Madore D, Chang I, Kohberger R, Watson W, Austrian R, Edwards K: Efficacy, safety and immunogenicity of heptavalent pneumococcal conjugate vaccine in children. Northern California Kaiser Permanente Vaccine Study Center Group. Pediatr Infect Dis J 2000, 19:187-195.

25. O'Brien KL, Moulton LH, Reid R, Weatherholtz R, Oski J, Brown L, Kumar G, Parkinson A, Hu D, Hackell J, Chang I, Kohberger R, Siber G, Santosham M: Efficacy and safety of seven-valent conjugate pneumococcal vaccine in American Indian children: group randomised trial. Lancet 2003, 362:355-361.

26. Black $S$, Shinefield $H$, Baxter $R$, Austrian $R$, Bracken $L$, Hansen J, Lewis $E$, Fireman B: Post licensure surveillance for pneumococcal invasive disease after use of heptavalent pneumococcal conjugate vaccine in Northern California Kaiser Permanente. Pediatr Infect Dis J 2004, 23:485-489.

27. Vestrheim DF, Lovoll O, Aaberge IS, Caugant DA, Høiby EA, Bakke $H$, Bergsaker MR: Effectiveness of a 2+1 dose schedule pneumococcal conjugate vaccination programme on invasive pneumococcal disease among children in Norway. Vaccine 2008, 26:3277-3281.

28. Eskola J, Kilpi T, Palmu A, Jokinen J, Haapakoski J, Herva E, Takala A, Kayhty H, Karma P, Kohberger R, Siber G, Makela PH, Finnish Otitis Media Study Group: Efficacy of a pneumococcal conjugate vaccine againstacute otitis media. N Engl J Med 2001, 344:403-409.

29. Hicks LA, Harrison LH, Flannery B, Hadler JL, Schaffner W, Craig AS, Jackson D, Thomas A, Beall B, Lynfield R, Reingold A, Farley MM, Whitney CG: Incidence of pneumococcal disease due to nonpneumococcal conjugate vaccine (PCV-7) serotypes in the United States during the era of widespread PCV-7 vaccination, 1998-2004. J Infect Dis 2007, 196:1346-1354.

30. Nigrovic LE, Kuppermann N, Malley R: Bacterial Meningitis Study Group of the Pediatric Emergency Medicine Collaborative Research Committee of the American Academy of Pediatrics: Children with bacterial meningitis presenting to the emergency department during the pneumococcal conjugate vaccine era. Acad Emerg Med 2008, 15:522-528.

31. Prymula R, Peeters P, Chrobok V, Kriz P, Novakova E, Kaliskova E, Kohl I, Lommel P, Poolman J, Prieels JP, Schuerman L: Pneumococcal capsular polysaccharides conjugated to protein $D$ for prevention of acute otitis media caused by both Streptococcus pneumonia and non-typable Haemophilus influenza: a randomised double-blind efficacy study. Lancet 2006, 367:740-748

32. Danovaro-Holiday MC, Garcia S, DeQuadros C, Tambini G, Andrus JK: Progress in Vaccination against Haemophilus influenzae type $b$ in the Americas. PLoS Med 2008, 5(4):e87.

33. Horn VK, Toth C, Wegienek J: Viability of microorganisms in four swab systems. ASM 98th General Meeting 1998, C436. 
34. National Committee for Clinical Laboratory Standards: Performance Standards for antimicrobial susceptibility testing. M100 S19. National Committee for Clinical Laboratory Standards Villanova, Pennsylvania; 2002.

35. Organización Panamericana de la Salud Programa de vigilancia de los serotipos y resistencia antimicrobiana de Streptococcus pneumoniae y Haemophilus influenzae Manual de procedimientos del proyecto SIREVA II. .

36. Commisso R, Romero-Orellano F, Montanaro PB, Romero-Moroni F, RomeroDiaz R: Acute otitis media: Bacteriology and bacterial resistance in 205 pediatric patients. Int J Pediatr Otorhinolaryngol 2000, 56:23-31.

37. Arguedas A, Loaiza C, Perez A, Vargas F, Herrera M, Rodriguez G, Gutierrez A, Mohs E: Microbiology of acute otitis media in Costa Rican children. Pediatr Infect Dis J 1998, 17:680-689.

38. Parras MM, Martinez AG, Echaniz-Aviles G, Lazcano-Ponce E, Apolinar YC, Colindres R, Hausdorff WP: Bacterial etiology and serotypes of acute otitis media in children in Mexic [Abstract no: A-181-0010-00611]. Buenos Aires, Argentina: Presented at 6th World Congress of the World Society for Pediatric Infectious Diseases (WSPID); 2009, Source: MIS (5564557).

39. Sierra A, Lopez P, Zapata MA, Vanegas B, Castrejon MM, DeAntonio R, Hausdorff WP, Colindres RE: Non-typeable Haemophilus influenza and Streptococcus pneumonia as primary causes of acute otitis media in Colombian children: a prospective study. BMC Infect Dis 2010, 11:1-11.

40. Pichichero ME, Casey JR, Hoberman A, Schwartz R: Pathogens causing recurrent and difficult-to-treat acute otitis media, 2003-2006. Clin Pediatr (Phila) 2008, 47:901-906

41. Block SL, Hedrick J, Harrison CJ, Tyler R, Smith A, Findlay R, Keegan E: Community-wide vaccination with the heptavalent pneumococcal conjugate significantly alters the microbiology of acute otitis media. Pediatr Infect Dis J 2004, 23:829-833.

42. McEllistrem MC, Adams JM, Patel K, Mendelsohn AB, Kaplan SL, Bradley JS, Schutze GE, Kim KS, Mason EO, Wald ER: Acute otitis media due to penicillin-nonsusceptible Streptococcus pneumonia before and after the introduction of the pneumococcal conjugate vaccine. Clin Infect Dis 2005, 40:1738-1744

43. Murphy TF, Faden H, Bakaletz LO, Kyd JM, Forsgren A, Campos J, Virji M, Pelton SI: Nontypeable Haemophilus influenza as a pathogen in children. Pediatr Infect Dis J 2009, 28:43-48.

44. Rodgers GL, Arguedas A, Cohen R, Dagan R: Global serotype distribution among Streptococcus pneumonia isolates causing otitis media in children: Potential implications for pneumococcal conjugate vaccines. Vaccine 2009, 27:3802-3810

45. Bardach A, Ciapponi A, Garcia-Marti S, Glujovsky D, Mazzoni A, Fayad A, Colindres RE: Gentile A Epidemiology of acute otitis media in children of Latin America and the Caribbean: A systematic review and metaanalysis. Int J Pediatr Otorhinolaryngol 2011, doi:10.1016/j.ijporl.2011.05.014.

46. Dabernat H, Seguy M, Faucon G, Delmas C: Epidemiology of Haemophilus influenzae strains collected in 2004 in France and in vitro assessment of their susceptibility to antibiotic. Médecine et maladies infectieuses 2007, 37:320-324.

47. Huang SS, Hinrichsen VL, Stevenson AE, Rifas-Shiman SL, Kleinman K, Pelton SI, Lipsitch M, Hanage WP, Lee GM, Finkelstein JA: Continued impact of pneumococcal conjugate vaccine on carriage in young children. Pediatrics 2009, 124:e1-e11.

48. Broides A, Dagan R, Greenberg D, Givon-Lavi N, Leibovitz E: Acute otitis media caused by Moraxella catarrhali: Epidemiologic and Clinical Characteristics. Clin Infect Dis 2009, 49:1641-1647.

49. Beekmann SE, Heilmann KP, Richter SS, Garcia-de-Lomas J, Doern GV: GRASP Study Group: Antimicrobial resistance in Streptococcus pneumonia, Haemophilus influenza, Moraxella catarrhali and group A beta-haemolytic streptococci in 2002-2003. Results of the multinational GRASP Surveillance Program. Int J Antimicrob Agents 2005, 25:148-156.

50. Piglansky L, Leibovitz E, Raiz S, Greenberg D, Press J, Leiberman A, Dagan R: Bacteriologic and clinical efficacy of high dose amoxicillin for therapy of acute otitis media in children. Pediatr Infect Dis J 2003, 22:405-413.

51. Whitney CG, Pilishvili T, Farley MM, Schaffner W, Craig AS, Lynfield R, Nyquist AC, Gershman KA, Vazquez M, Bennett NM, Reingold A, Thomas A, Glode MP, Zell ER, Jorgensen JH, Beall B, Schuchat A: Effectiveness of seven-valent pneumococcal conjugate vaccine against invasive pneumococcal disease: a matched case-control study. Lancet 2006, 368:1495-1502.
52. Hausdorff WP, Hoet B, Schuerman L: Do pneumococcal conjugate vaccines provide any cross-protection against serotype 19A? BMC Pediatr 2010, 10:1-7.

\section{Pre-publication history}

The pre-publication history for this paper can be accessed here: http://www.biomedcentral.com/1471-2334/12/40/prepub

doi:10.1186/1471-2334-12-40

Cite this article as: Naranjo et al:: Non-capsulated and capsulated Haemophilus influenzae in children with acute otitis media in Venezuela: a prospective epidemiological study. BMC Infectious Diseases 2012 12:40.

\section{Submit your next manuscript to BioMed Central and take full advantage of:}

- Convenient online submission

- Thorough peer review

- No space constraints or color figure charges

- Immediate publication on acceptance

- Inclusion in PubMed, CAS, Scopus and Google Scholar

- Research which is freely available for redistribution 